\title{
Disjointness Preserving and Functional Type Disjointness Preserving Operators
}

\author{
C. Ganesa Moorthy ${ }^{1}$ and C. T. Ramasamy ${ }^{2}$ \\ ${ }^{1}$ Department of Mathematics, Alagappa University, Karaikudi 630 003, India \\ ${ }^{2}$ Department of Mathematics, H. H. The Rajah's College, Pudukkottai, Tamil Nadu 622001, India \\ Correspondence should be addressed to C. T. Ramasamy; ctrams83@gmail.com
}

Received 19 November 2013; Accepted 5 January 2014; Published 25 February 2014

Academic Editors: Y. Latushkin and Z. Wu

Copyright ( 2014 C. G. Moorthy and C. T. Ramasamy. This is an open access article distributed under the Creative Commons Attribution License, which permits unrestricted use, distribution, and reproduction in any medium, provided the original work is properly cited.

\begin{abstract}
A new concept called functional type disjointness preserving operators is introduced and structure of disjointness preserving and functional type disjointness preserving operators on some function spaces are analysed.
\end{abstract}

\section{Introduction}

There are many articles for construction of unbounded disjointness preserving operators. The paper [1] of Abramovich and Lipecki develops techniques to construct unbounded disjointness preserving linear functionals on any infinite dimensional $F$-lattice, and this is also mentioned in Remark 2.7 of the paper [2]. This paper [2] is devoted to construct unbounded disjointness preserving linear functionals on function spaces $C_{0}(X)$, when $X$ is a locally compact Hausdorff space. The paper [3] of Jeang and Wong presents a simplified procedure to construct unbounded disjointness preserving functionals on function spaces $C_{0}(X)$. Jarosz [4] presents a construction of disjointness preserving operators on $C(X)$, where $X$ is a compact Hausdorff space. Disjointness preserving operators are defined on many structures, namely, lattices, function spaces, algebras, and so forth. The present paper is restricted to study disjointness preserving operators on function spaces. A linear operator $T$ between two function spaces is said to be disjointness preserving if $T f \cdot T g=0$ whenever $f \cdot g=0$. The second section of the paper exercises the standard techniques applicable to derive an essential structure of disjointness preserving operators. This is done on the function spaces $C_{c}(X), C_{0}(X)$ for a normal space $X$, and on $\mathscr{D}(\mathbb{R}), C_{0}^{\infty}(\mathbb{R})$. Although results hold for both real and complex cases, it is assumed that the results on $\mathscr{D}(\mathbb{R}), C_{0}^{\infty}(\mathbb{R})$ are over real fields.
For a disjointness preserving linear functional $T$ we have $T f=0$ or $T g=0$, whenever $f \cdot g=0$. Jarosz [4] constructed an unbounded disjointness preserving operator $T$ from $C(X)$ onto $E$, for any given infinite compact Hausdorff spaces $X$ and $Y$, and for any given linear subspace $E$ of $C(Y)$ such that $1 \leq \operatorname{dim} E \leq c$ (cardinality of continuum). These mappings have the following property: $f \cdot g=0$ implies $T f=0$ or $\mathrm{Tg}=0$. So, let us give a new name to this type of mappings.

Definition 1. A linear operator $T$ from a function space into a function space is said to be a functional type disjointness preserving (FTDP) if $f \cdot g=0$ implies $T f=0$ or $T g=0$.

An essential structure of FTDP-mappings is derived on some function spaces in Section 3.

For a real or complex valued function $f$ on $X$, we define the cozero set of $f$ by $\operatorname{coz}(f)=\{x \in X: f(x) \neq 0\}$, and zero set of $f$ by $z(f)=\{x \in X: f(x)=0\}$ and if $X$ is a topological space, then we define the support of $f$ by supp $f=\overline{\operatorname{coz}(f)}$, closure of cozero of $f$. For a nonempty set $Y, F(Y)$ will denote the linear space of all real or complex valued functions defined on $Y$. To each $y \in Y, \delta_{y}$ will denote the evaluation functional defined on $F(Y)$ by $\delta_{y}(f)=f(y)$, for every $f \in F(Y)$. All the topological spaces to be considered are Hausdorff spaces. For a topological space $X, C(X)$ will denote the linear space of all real or complex valued bounded continuous functions; $C_{c}(X)$ will denote the linear space of 
all real or complex valued continuous functions with compact support in $X ; C_{0}(X)$ will denote the linear space $C_{0}(X)=\{f$ : $X \rightarrow \mathbb{R}$ or $\mathbb{C}: f$ is continuous on $X$, and for every $\epsilon>0$ there is a compact subset $K$ of $X$ such that $|f(x)|<\epsilon$, for every $x \in X \backslash K\}$. Let us use the usual notation $C^{\infty}(\mathbb{R})$ to denote the linear space of all real valued functions defined on the real line $\mathbb{R}$ which are infinitely many times differentiable at every point in $\mathbb{R} . \mathscr{D}(\mathbb{R})$ will denote the linear space of all test functions on $\mathbb{R}$ (see [5]). That is, $\mathscr{D}(\mathbb{R})=\left\{f \in C^{\infty}(\mathbb{R}): f\right.$ has compact support in $\mathbb{R}\}$. Let us use the notation $C_{0}^{\infty}(\mathbb{R})$ to denote the linear space: $C_{0}^{\infty}(\mathbb{R})=\left\{f \in C^{\infty}(\mathbb{R})\right.$ : for every $\epsilon>0$, there is a compact subset $K$ of $\mathbb{R}$ such that $|f(x)|<\epsilon$, for all $x \in \mathbb{R} \backslash K\}$. We consider the linear spaces $C(X), C_{c}(X), C_{0}(X), \mathscr{D}(\mathbb{R})$, and $C_{0}^{\infty}(\mathbb{R})$ as normed spaces with the supremum norms on them. If $X$ is normal, then $C_{c}(X)$ is dense in $C_{0}(X)$. For if $f \in C_{0}(X)$ and $\epsilon>0$, then there is a $g \in C_{c}(X)$ such that $\|g\|=1,0 \leq g \leq 1, g$ has value 1 on $\{x \in X:|f(x)| \geq \epsilon\}, g$ has value 0 on $\{x \in X:|f(x)| \leq$ $\epsilon / 2\}, f \cdot g \in C_{c}(X)$, and $\|f \cdot g-f\| \leq \epsilon$. Similarly, $\mathscr{D}(\mathbb{R})$ is also dense subspace of $C_{0}^{\infty}(\mathbb{R})$, which follows from Lemma 1 of Section 1.8 in [6].

\section{Disjointness Preserving Mappings}

The following theorem is a variation of the results obtained by Jarosz [4] and by Jeang and Wong [3].

Theorem 2 (see $[3,4]$ ). Let $T$ be a linear disjointness preserving mapping from $C(X)$ (or $\left.C_{0}(X)\right)$ to $F(Y)$ where $X$ is a compact Hausdorff space (or locally compact Hausdorff space $X)$ and $Y$ is a nonempty set. Let

$$
\begin{aligned}
Y_{1}= & \{y \in Y: \\
& \left.\delta_{y} \circ T \text { is a nonzero continuous linear functional }\right\}, \\
Y_{2}= & \left\{y \in Y: \delta_{y} \circ T \text { is discontinuous }\right\}, \\
Y_{3}= & \left\{y \in Y: \delta_{y} \circ T=0\right\} .
\end{aligned}
$$

Then there is a function $\phi: Y_{1} \cup Y_{2} \rightarrow X\left(\operatorname{or} \phi: Y_{1} \cup Y_{2} \rightarrow\right.$ $X_{\infty}, X_{\infty}=X \cup\{\infty\}$ is the one point compactification of $\left.X\right)$ such that $T f(y)=\alpha_{y} f(\phi(y))$ for all $f \in C(X)\left(\right.$ or $\left.C_{0}(X)\right)$, for some fixed scalar $\alpha_{y}$, and for all $y \in Y_{1}$ such that $\delta_{y} \circ T=0$ on $\left\{f \in C(X)\left(\operatorname{or} C_{0}(X)\right): \phi(y) \notin \operatorname{supp} f\right\}$, for all $y \in Y_{1} \cup$ $Y_{2}$. Moreover, if every function in the range of $T$ is a bounded function on $Y$, then $\phi\left(Y_{2}\right)$ is a finite subset of $X$ (or $X_{\infty}$, resp., and $\phi(y) \in X=X_{\infty} \backslash\{\infty\}$ for $\left.y \in Y_{1}\right)$.

These results depend on the Urysohn lemma and existence of partitions of unity. Let us also use them in deriving the following theorem.

Theorem 3. Let $X$ be a normal space and $Y$ be a nonempty set. Let $T$ be a linear disjointness preserving mapping from $C_{c}(X)$ into $F(Y)$. Let $Y_{1}, Y_{2}$, and $Y_{3}$ be defined as in Theorem 2. Then there is a function $\phi: Y_{1} \cup Y_{2} \rightarrow X$ such that $\delta_{y} \circ T=\alpha_{y} \delta_{\phi(y)}$ on $C_{c}(X)$ for some fixed scalar $\alpha_{y}$, and for all $y \in Y_{1}$ such that $\delta_{y} \circ T=0$ on $\{f \in C(X): \phi(y) \notin \operatorname{supp} f\}$, for all $y \in Y_{1} \cup Y_{2}$.
Proof. To each $y \in Y$, define $\Phi(y)=\{x \in X$ : for every open neighbourhood $U$ of $x$, there is an $f \in C_{c}(X)$ such that $\operatorname{coz}(f) \subset U$ and $T f(y) \neq 0\}$. First, we claim that to each $y \in Y, \Phi(y)$ contains at most one point. On the contrary, suppose $x_{1}, x_{2} \in \Phi(y)$ for some $y \in Y$, with $x_{1} \neq x_{2}$. Let $U_{1}$ and $U_{2}$ be two disjoint open neighbourhoods of $x_{1}$ and $x_{2}$ respectively. Then there are $f_{1}, f_{2} \in C_{c}(X)$ such that $T f_{i}(y) \neq 0$ and $\operatorname{coz}\left(f_{i}\right) \subset U_{i}, i=1,2$. This is a contradiction to the assumption that $T$ is disjointness linear preserving. So, $\Phi(y)$ contains at most one point, for every $y \in Y$. We next claim that $\Phi(y)$ is nonempty for every $y \in Y_{1} \cup Y_{2}$. On the contrary we assume that $\Phi(y)$ is empty for some $y \in Y_{1} \cup Y_{2}$. Note that $\delta_{y} \circ T \neq 0$. Fix $f \in C_{c}(X)$. Let $K=\operatorname{supp} f$. To each $x \in K$, let $U_{x}$ be an open neighbourhood of $x$ such that $\operatorname{Tg}(y)=0$ whenever $g \in C_{c}(X)$ and $\operatorname{coz}(g) \subset U_{x}$. Then we can find a finite subfamily $\left\{U_{1}, U_{2}, \ldots, U_{n}\right\}$ of $\left\{U_{x}: x \in X\right\}$ such that the subfamily covers $K$. Let $\left\{g_{1}, g_{2}, \ldots, g_{n}\right\} \subset C(K)$ be a continuous decomposition of the identity subordinate to $\left\{U_{1} \cap K, U_{2} \cap K, \ldots, U_{n} \cap K\right\}$. Then, by the Tietze extension theorem, there are functions $f_{1}, f_{2}, \ldots, f_{n}$ in $C(X)$ such that $f_{i \mid K}=g_{i}$ and $0 \leq f_{i} \leq 1$, for every $i$. Then $f \cdot f_{i} \in C_{c}(X)$ and $\operatorname{coz}\left(f \cdot f_{i}\right) \subset U_{i}$ and hence $T\left(f \cdot f_{i}\right)(y)=0$, for every i. So, $T f(y)=T\left(\sum_{i=1}^{n} f \cdot f_{i}\right)(y)=\sum_{i=1}^{n} T\left(f \cdot f_{i}\right)(y)=0$. Thus $\delta_{y} \circ T=0$ on $C_{c}(X)$, which is a contradiction. Hence $\Phi(y)$ is a singleton set for each $y \in Y_{1} \cup Y_{2}$. Define a function $\phi: Y_{1} \cup Y_{2} \rightarrow X$ by $\{\phi(y)\}=\Phi(y)$. To each $y \in Y_{1} \cup Y_{2}$, write $J_{y}=\left\{f \in C_{c}(X): \phi(y) \notin \operatorname{supp} f\right\}$, and $K_{y}=\left\{f \in C_{c}(X):\right.$ $f(\phi(y))=0\}$. If $y \in Y_{1} \cup Y_{2}$ and $f \in J_{y}$, then $\phi(y) \notin \operatorname{supp} f$ and there is a $g \in C_{c}(X)$ with $\operatorname{coz}(g) \subset X \backslash$ supp $f$ such that $\operatorname{Tg}(y) \neq 0$. Since $T$ is disjointness preserving, $T f(y)=0$. Thus $J_{y} \subset \operatorname{ker}\left(\delta_{y} \circ T\right)$. Since $X$ is normal, if $f \in K_{y}, y \in Y_{1} \cup Y_{2}$, and $\epsilon>0$, then there is a $g \in C_{c}(X)$ such that $\|g\|=1$, $0 \leq g \leq 1, g$ has value 1 on $\{x \in X:|f(x)| \geq \epsilon\}$, and $g$ has value 0 on $\{x \in X:|f(x)| \leq \epsilon / 2\}, f \cdot g \in C_{c}(X), f \cdot g \in J_{y}$, and $\|f \cdot g-f\| \leq \epsilon$. Thus $J_{y}$ is dense in $K_{y}$. If $y \in Y_{1}$, then $\operatorname{ker}\left(\delta_{y} \circ T\right)$ is closed and hence $K_{y} \subset \operatorname{ker}\left(\delta_{y} \circ T\right)$. So $K_{y}=\operatorname{ker}\left(\delta_{y} \circ T\right)$ and $\delta_{y} \circ T=\alpha_{y} \delta_{\phi(y)}$ on $C_{c}(X)$, for some $\alpha_{y}$. This proves the theorem.

Corollary 4. Let $X$ be a normal space and $Y$ a nonempty set. Let $T$ be a linear disjointness preserving mapping from $C_{0}(X)$ to $F(Y)$. Suppose $\delta_{y} \circ T$ is continuous on $C_{0}(X)$, for every $y \in Y$. Let

$$
\begin{aligned}
Y_{1}=\{y \in Y: & \\
& \left.\delta_{y} \circ T \text { is a nonzero continuous linear functional }\right\},
\end{aligned}
$$$$
Y_{2}=\left\{y \in Y: \delta_{y} \circ T=0\right\}
$$

Then there is a function $\phi: Y_{1} \rightarrow X$ such that $\delta_{y} \circ T=\alpha_{y} \delta_{\phi(y)}$ on $C_{0}(X)$ for some fixed scalar $\alpha_{y}$ and for all $y \in Y_{1}$.

Proof. Consider the restriction of $T$ to $C_{c}(X)$. Then we conclude that $\delta_{y} \circ T=\alpha_{y} \delta_{\phi(y)}$ on $C_{c}(X)$, for some fixed scalar $\alpha_{y}$, because $\delta_{y} \circ T=0$ on $C_{c}(X)$ if and only if $\delta_{y} \circ T=0$ on $C_{0}(X)$. Continuity assumption on $\delta_{y} \circ T$ also implies that the relation $\delta_{y} \circ T=\alpha_{y} \delta_{\phi(y)}$ is true on $C_{0}(X)$ also because $C_{c}(X)$ is dense in $C_{0}(X)$. 
The classical Urysohn lemma and the result on existence of partitions of unity have their version in $\mathscr{D}(\mathbb{R})$ (see: [6, Section 1.8 Lemma 1] and [5, Theorem 6.20]). So we have the following version of the previous theorem to the space $\mathscr{D}(\mathbb{R})$.

Theorem 5. Let $Y$ be a nonempty set. Let $T$ be a linear disjointness preserving mapping from $\mathscr{D}(\mathbb{R})$ to $F(Y)$. Let $Y_{1}$, $Y_{2}$, and $Y_{3}$ be defined as in Theorem 2. Then there is a function $\phi: Y_{1} \cup Y_{2} \rightarrow \mathbb{R}$ such that $\delta_{y} \circ T=\alpha_{y} \delta_{\phi(y)}$ on $\mathscr{D}(\mathbb{R})$ for some fixed scalar $\alpha_{y}$ and for all $y \in Y_{1}$ and $\delta_{y} \circ T=0$ on $\{f \epsilon$ $\mathscr{D}(\mathbb{R}): \phi(y) \notin \operatorname{supp} f\}$, for all $y \in Y_{1} \cup Y_{2}$.

Proof. To each $y \in Y$, define $\Phi(y)=\{x \in \mathbb{R}$ : for every open neighbourhood $U$ of $x$, there is an $f \in \mathscr{D}(\mathbb{R})$ such that $\operatorname{coz}(f) \subset U$ and $T f(y) \neq 0\}$. It is easy to verify that $\Phi(y)$ contains at most one point, for every $y \in Y$. We next claim that $\Phi(y)$ is nonempty for every $y \in Y_{1} \cup Y_{2}$. On the contrary we assume that $\Phi(y)$ is empty for some $y \in Y_{1} \cup Y_{2}$. Note that $\delta_{y} \circ T \neq 0$. Fix $f \in \mathscr{D}(\mathbb{R})$. Let $K=\operatorname{supp} f$. To each $x \in K$, let $U_{x}$ be an open neighbourhood of $x$ such that $\operatorname{Tg}(y)=0$ whenever $g \in \mathscr{D}(\mathbb{R})$ and $\operatorname{coz}(g) \subset U_{x}$. Then we can find a finite subfamily $\left\{U_{1}, U_{2}, \ldots, U_{n}\right\}$ of $\left\{U_{x}: x \in X\right\}$ such that the subfamily covers $K$. By [5, Theorem 6.20] there are functions $f_{1}, f_{2}, \ldots, f_{n}$ in $\mathscr{D}(\mathbb{R})$ such that $0 \leq f_{i} \leq 1$, for every $i$, $f_{1}+f_{2}+\cdots+f_{n}=1$ on $K$ and $\operatorname{supp} f_{i} \subset U_{i}$, for all $i$. Then $f \cdot f_{i} \in \mathscr{D}(\mathbb{R})$ and $\operatorname{coz}\left(f \cdot f_{i}\right) \subset U_{i}$ and hence $T\left(f \cdot f_{i}\right)(y)=0$, for every $i$. So, $T f(y)=T\left(\sum_{i=1}^{n} f \cdot f_{i}\right)(y)=\sum_{i=1}^{n} T\left(f \cdot f_{i}\right)(y)=$ 0 . Thus $\delta_{y} \circ T=0$ on $\mathscr{D}(\mathbb{R})$, which is a contradiction. Hence $\Phi(y)$ is a singleton set for each $y \in Y_{1} \cup Y_{2}$. Define a function $\phi: Y_{1} \cup Y_{2} \rightarrow \mathbb{R}$ by $\{\phi(y)\}=\Phi(y)$. To each $y \in Y_{1} \cup Y_{2}$, write $J_{y}=\{f \in \mathscr{D}(\mathbb{R}): \phi(y) \notin \operatorname{supp} f\}$, and $K_{y}=\{f \in \mathscr{D}(\mathbb{R})$ : $f(\phi(y))=0\}$. If $y \in Y_{1} \cup Y_{2}$ and $f \in J_{y}$, then $\phi(y) \notin \operatorname{supp} f$ and there is a $g \in \mathscr{D}(\mathbb{R})$ with $\operatorname{coz}(g) \subset X \backslash$ supp $f$ such that $\operatorname{Tg}(y) \neq 0$. Since $T$ is disjointness preserving, $T f(y)=0$. Thus $J_{y} \subset \operatorname{ker}\left(\delta_{y} \circ T\right)$. If $f \in K_{y}, y \in Y_{1} \cup Y_{2}$, and $\epsilon>0$, then by $[6$, Lemma 1 of Section 1.8] there is a $g \in \mathscr{D}(\mathbb{R})$ such that $\|g\|=1,0 \leq g \leq 1, g$ has value 1 on $\{x \in \mathbb{R}:|f(x)| \geq \epsilon\}$, and $g$ has value 0 on $\{x \in \mathbb{R}:|f(x)| \leq \epsilon / 2\}, f \cdot g \in J_{y}$, and $\|f \cdot g-f\| \leq \epsilon$. Thus $J_{y}$ is dense in $K_{y}$. If $y \in Y_{1}$, then $\operatorname{ker}\left(\delta_{y} \circ T\right)$ is closed and hence $K_{y} \subset \operatorname{ker}\left(\delta_{y} \circ T\right)$. So $K_{y}=\operatorname{ker}\left(\delta_{y} \circ T\right)$ and $\delta_{y} \circ T=\alpha_{y} \delta_{\phi(y)}$ on $\mathscr{D}(\mathbb{R})$, for some $\alpha_{y}$. This proves the theorem.

Observe that $\mathscr{D}(\mathbb{R})$ is dense in $C_{0}^{\infty}(\mathbb{R})$ in view of $[6$, Lemma 1 in Section 1.8]. So, we have the following corollary.

Corollary 6. Let $Y$ be a nonempty set. Let $T$ be a linear disjointness preserving mapping from $C_{0}^{\infty}(\mathbb{R})$ to $F(Y)$. Suppose $\delta_{y} \circ T$ is continuous on $C_{0}^{\infty}(\mathbb{R})$, for every $y \in Y$. Let $Y_{1}, Y_{2}$ be defined as in Corollary 4. Then there is a function $\phi: Y_{1} \rightarrow X$ such that $\delta_{y} \circ T=\alpha_{y} \delta_{\phi(y)}$ on $C_{0}^{\infty}(\mathbb{R})$ for some fixed scalar $\alpha_{y}$, and for all $y \in Y_{1}$.

Remark 7. One may change $\mathbb{R}$ into any open region in $\mathbb{R}^{n}$ in Theorem 5 and Corollary 6. $C_{0}^{\infty}(\mathbb{R})$ may be replaced in Corollary 6 by any of its linear subspace containing $\mathscr{D}(\mathbb{R})$. $C_{0}(X)$ may be replaced in Corollary 4 by any of its linear subspace containing $C_{c}(X)$.

\section{FTDP-Mappings}

Theorem 8. Let $X$ be a compact Hausdorff space and $Y$ a nonempty set. Let $T: C(X) \rightarrow F(Y)$ be a nonzero disjointness preserving mappings. Let $\phi, Y_{1}, Y_{2}$, and $Y_{3}$ be defined as in Theorem 2. Consider the following statements.

(i) $T$ is a FTDP-mapping.

(ii) There is a unique $x_{0} \in X$ such that, for every open neighbourhood $U$ of $x_{0}$, there is a $f \in C(X)$ with $\operatorname{coz}(f) \subset U$ such that $T f \neq 0$ in $F(Y)$.

(iii) $\phi\left(Y_{1} \cup Y_{2}\right)$ is a singleton subset of $X$.

(iv) $\cap\{\operatorname{supp} f: f \in C(X), T f \neq 0$ in $F(Y)\}$ is a singleton set.

Then the implications (i) $\Rightarrow($ ii), $\quad(i) \Rightarrow($ iii $), \quad(i) \Rightarrow(i v)$, and (iii) $\Rightarrow$ (ii) are true. If ( $i)$ is true, then the singleton sets in (iii) and (iv) are $\left\{x_{0}\right\}$ for $x_{0}$ given in (ii). If (ii) is true, then $x_{0} \in$ $\overline{\phi\left(Y_{1} \cup Y_{2}\right)}$.

Proof. (i) $\Rightarrow$ (ii): Suppose $T$ is a FTDP-mapping. Suppose there are two distinct points $x_{1}, x_{2} \in X$ with the property given in (ii). We can find two disjoint open neighbourhoods $U_{1}$, $U_{2}$ of $x_{1}, x_{2}$, respectively. Then there are $f_{1}, f_{2} \in C(X)$ with $\operatorname{coz}\left(f_{1}\right) \subset U_{1}, \operatorname{coz}\left(f_{2}\right) \subset U_{2}$ such that $T f_{1} \neq 0$ and $T f_{2} \neq 0$. This is a contradiction. So, uniqueness of $x_{0}$ in (ii) is established. If there is no $x_{0}$ in $X$ with the property mentioned in (ii), then for each $x \in X$, there is a neighbourhood $U_{x}$ of $x$ such that $T f=0$ in $F(Y)$ whenever $\operatorname{coz}(f) \subset U_{x}$. Then we find a subcover $\left\{U_{1}, U_{2}, \ldots, U_{n}\right\}$ of $\left\{U_{x}: x \in X\right\}$ for $X$. Then we find a partition $g_{1}, g_{2}, \ldots, g_{n}$ for unity in $C(X)$ such that $\operatorname{coz}\left(g_{i}\right) \subset U_{i}, i=1,2, \ldots, n$. Then for every $f \in C(X)$, we have $T f=T\left(\sum_{i=1}^{n} f g_{i}\right)=\sum_{i=1}^{n} T\left(f g_{i}\right)=0$. Thus $T=0$ on $C(X)$, a contradiction. So (ii) is true.

Note that $\phi\left(Y_{1} \cup Y_{2}\right)$ is nonempty, because $T \neq 0$ on $C(X)$. Moreover $y \in Y_{1} \cup Y_{2}$ if and only if $\delta_{y} \circ T \neq 0$ on $C(X)$. We claim that $x_{0} \in \overline{\phi\left(Y_{1} \cup Y_{2}\right)}$. On the contrary, we assume that $x_{0} \notin \overline{\phi\left(Y_{1} \cup Y_{2}\right)}$. Find an open neighbourhood $U_{0}$ of $x_{0}$ and a function $f_{0} \in C(X)$ such that $\operatorname{coz}\left(f_{0}\right) \subset U_{0}, T f_{0} \neq 0$ in $F(Y)$, and $\bar{U}_{0} \cap \overline{\phi\left(Y_{1} \cup Y_{2}\right)}=\emptyset$. Fix any $y \in Y_{1} \cup Y_{2}$. Let $x_{1}=\phi(y)$. Then there is an open neighbourhood $U_{1}$ of $x_{1}$ such that $\bar{U}_{0} \cap$ $U_{1}=\emptyset$. Find a function $f_{1} \in C(X)$ such that $\operatorname{coz}\left(f_{1}\right) \subset U_{1}$ and $T f_{1}(y) \neq 0$ in $C(X)$. So $T f_{0}(y)=0$, because $T$ is disjointness preserving. Thus $T f_{0}(y)=0$, for any $y \in Y_{1} \cup Y_{2}$. So $T f_{0}=$ 0 , where $T f_{0} \neq 0$ in $C(X)$. Therefore, we conclude that $x_{0} \in$ $\overline{\phi\left(Y_{1} \cup Y_{2}\right)}$, if (ii) is true.

(i) $\Rightarrow$ (iii): Suppose $T$ is a FTDP-mapping. Since $T \neq 0, Y_{1} \cup$ $Y_{2}$ is nonempty. Suppose $\phi\left(y_{1}\right) \neq \phi\left(y_{2}\right)$ for some $y_{1}, y_{2} \in Y_{1} \cup$ $Y_{2}$. Find two disjoint open sets $U$ and $V$ such that $\phi\left(y_{1}\right) \in U$ and $\phi\left(y_{2}\right) \in V$. Find $f, g \in C(X)$ such that $\operatorname{coz}(f) \subset U$, $\operatorname{coz}(g) \subset V$, and $T f\left(y_{1}\right) \neq 0, T g\left(y_{2}\right) \neq 0$. Then $f \cdot g=0$ in $C(X)$, but $T f \neq 0$ and $T g \neq 0$ in $F(Y)$. This is a contradiction. So, $\phi\left(Y_{1} \cup Y_{2}\right)$ is a singleton set, and $\phi\left(Y_{1} \cup Y_{2}\right)=\overline{\phi\left(Y_{1} \cup Y_{2}\right)}$.

(i) $\Rightarrow$ (iv): Suppose (i) is true. Consider $x_{0}$ given in (ii). Suppose $f \in C(X), T f \neq 0$ in $F(Y)$, and $x_{0} \notin$ supp $f$. Then find $g \in C(X)$ such that $\operatorname{coz}(g) \subset X \backslash$ supp $f$ and $T g \neq 0$ in $F(Y)$. Thus $f \cdot g=0, T f \neq 0$ and $T g \neq 0$, a contradiction. Thus $x_{0} \in \operatorname{supp} f$ if $T f \neq 0$. If $x_{1} \in X$ and $x_{1} \neq x_{0}$, find two disjoint 
open neighbourhoods $U_{0}$ and $U_{1}$ of $x_{0}$ and $x_{1}$, respectively. Find $f \in C(X)$ such that $T f \neq 0$ and $\operatorname{coz}(f) \subset U_{0}$. Then $x_{1} \notin \operatorname{supp} f$. So, $\left\{x_{0}\right\}=\cap\{\operatorname{supp} f: f \in C(X), T f \neq 0$ in $F(Y)\}$, where $x_{0}$ the member mentioned in (ii). This proves (iv).

(iii) $\Rightarrow$ (ii): Suppose (iii) is true, and suppose $\phi\left(Y_{1} \cup Y_{2}\right)=$ $\left\{x_{0}\right\}$. Then for every $y \in Y_{1} \cup Y_{2}$ and for every open neighbourhood $U$ of $x_{0}$ there is a function $f \in C(X)$ such that $\operatorname{coz}(f) \subset U$ and $T f(y) \neq 0$, and hence $T f \neq 0$ in $F(Y)$. Let $x_{1} \in$ $X$ be such that $x_{1} \neq x_{0}$. Find two disjoint neighbourhoods of $U_{0}, U_{1}$ of $x_{0}, x_{1}$, respectively. Fix $y \in Y_{1} \cup Y_{2}$ arbitrarily. Find a function $f \in C(X)$ such that $\operatorname{coz}(f) \subset U_{0}$ and $T f(y) \neq 0$. Then for every function $g \in C(X)$ with $\operatorname{coz}(g) \subset U_{1}$, we have $f \cdot g=0$, and hence $\operatorname{Tg}(y)=0$, since $T$ is disjointness preserving. Thus for every function $g \in C(X)$ with $\operatorname{coz}(g) \subset$ $U_{1}$, we have $\operatorname{Tg}(y)=0$, for every $y \in Y_{1} \cup Y_{2}$, and hence we have $T g=0$ in $F(Y)$. This of course proves (ii), and it is proved that $x_{0}$ is the member mentioned in (ii).

Corollary 9. Let $X$ be a compact Hausdorff space and $Y$ be a nonempty set. Let $T: C(X) \rightarrow F(Y)$ a nonzero FTDP-mapping. Then the following are equivalent.

(i) $\delta_{y} \circ T$ is continuous for every $y \in Y$.

(ii) $T f=\delta_{x_{0}}(f) g$ on $C(X)$ for some fixed function $g \in$ $F(Y)$, for some fixed $x_{0} \in X$, and for all $f \in C(X)$.

Proof. Suppose (i) is true. Suppose $\phi\left(Y_{1} \cup Y_{2}\right)=\left\{x_{0}\right\}$. Then for every $y \in Y_{1} \cup Y_{2}$, we have $\left\{f \in C(X): x_{0} \notin \operatorname{supp} f\right\} \subset$ $\operatorname{ker}\left(\delta_{y} \circ T\right)$, and hence $\left\{f \in C(X): f\left(x_{0}\right)=0\right\}=\operatorname{ker}\left(\delta_{y} \circ T\right)$. Thus, $\operatorname{ker} T=\left\{f \in C(X): f\left(x_{0}\right)=0\right\}$. This proves (ii). Another implication is obvious.

Remark 10. Theorem 8 is extendable to $C_{c}(X)$ when $X$ is normal and to $\mathscr{D}(\mathbb{R})$. Corollary 9 is extendable to $C_{c}(X)$, $C_{0}(X), \mathscr{D}(\mathbb{R})$, and $C_{0}^{\infty}(\mathbb{R})$ when $X$ is normal. Theorem 8 is extendable to $C_{0}(X)$ when $X$ is locally compact with an additional assumption that the singleton set may be $\{\infty\}$ in $X_{\infty}$. Corollary 9 is extendable to $C_{0}(X)$ when $X$ is locally compact.

\section{Conflict of Interests}

The authors declare that there is no conflict of interests regarding the publication of this paper.

\section{References}

[1] Y. A. Abramovich and Z. Lipecki, "On ideals and sublattices in linear lattices and F-lattices," Mathematical Proceedings of the Cambridge Philosophical Society, vol. 108, no. 1, pp. 79-87, 1990.

[2] L. G. Brown and N. C. Wong, "Unbounded disjointness preserving linear functionals," Monatshefte fur Mathematik, vol. 141, no. 1, pp. 21-32, 2004.

[3] J. S. Jeang and N. C. Wong, "Weighted composition operators of $C_{0}(X)$ 's," Journal of Mathematical Analysis and Applications, vol. 201, no. 3, pp. 981-993, 1996.

[4] K. Jarosz, "Automatic continuity of separating linear isomorphisms," Canadian Mathematical Bulletin, vol. 33, pp. 139-1144, 1990.
[5] W. Rudin, Functional Analysis, International Series in Pure and Applied Mathematics, McGraw-Hill, New York, NY, USA, 2nd edition, 1991.

[6] A. H. Zemanian, Distribution Theory and Tranform Analysis: An Introduction to Generalized Functions with Applications, Dover, New York, NY, USA, 1987. 


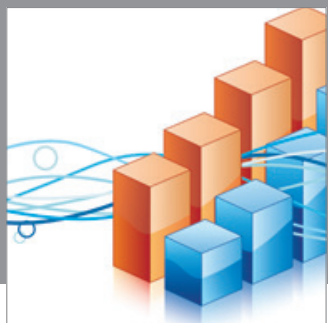

Advances in

Operations Research

mansans

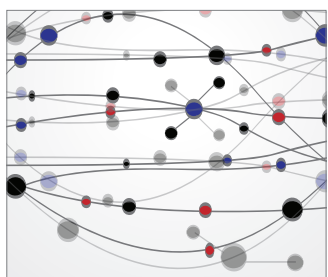

The Scientific World Journal
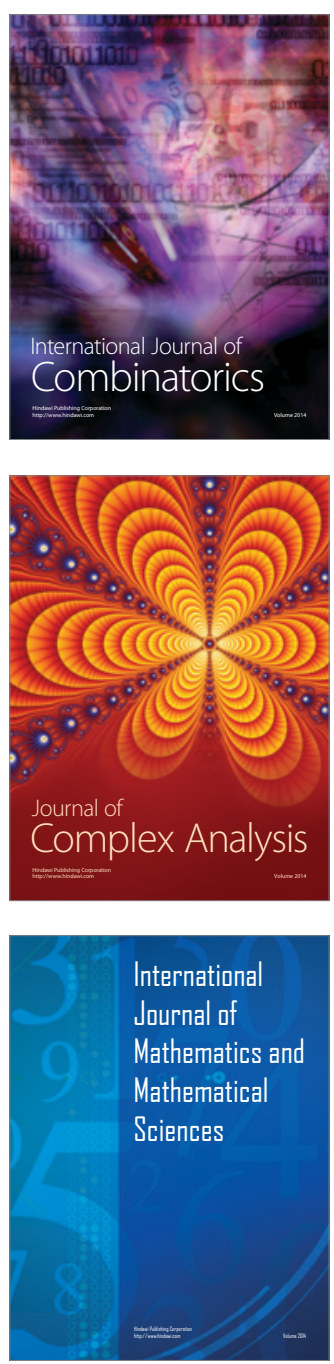
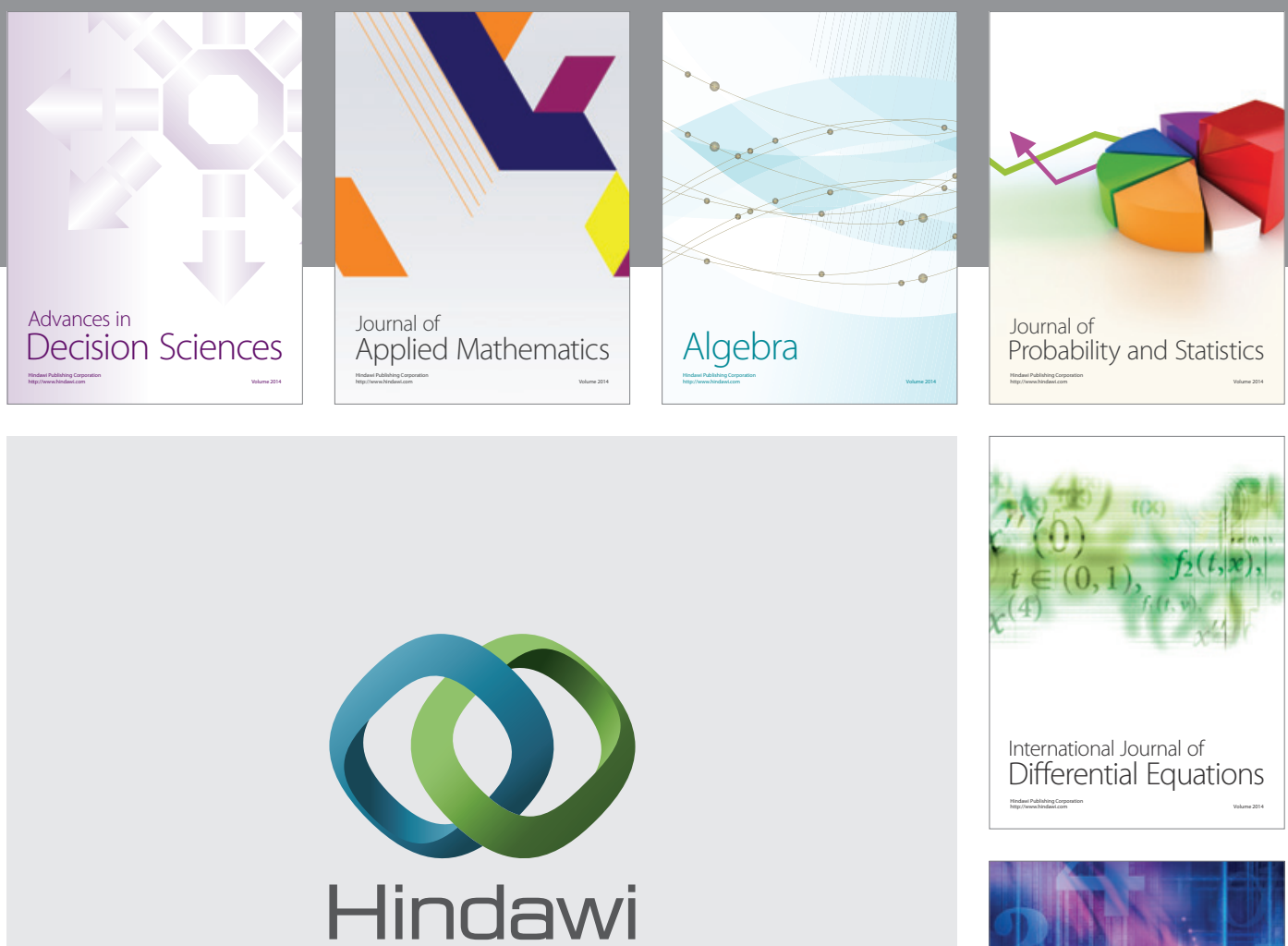

Submit your manuscripts at http://www.hindawi.com
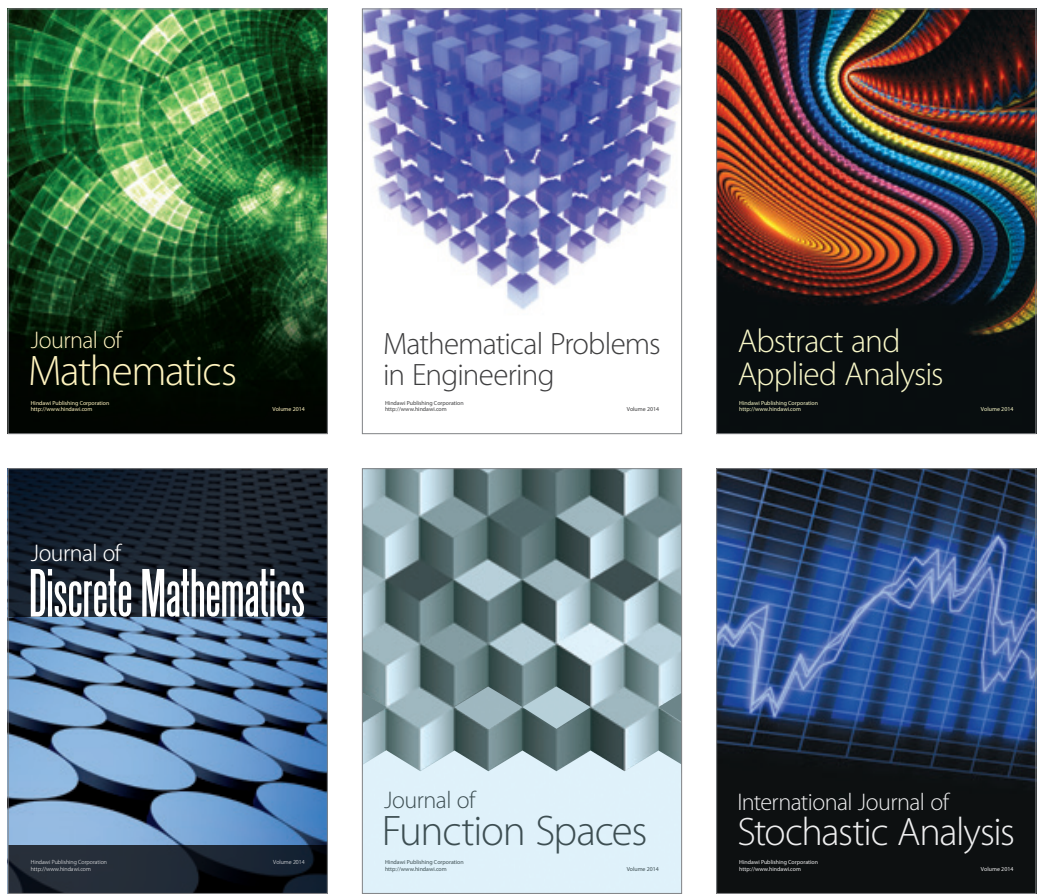

Journal of

Function Spaces

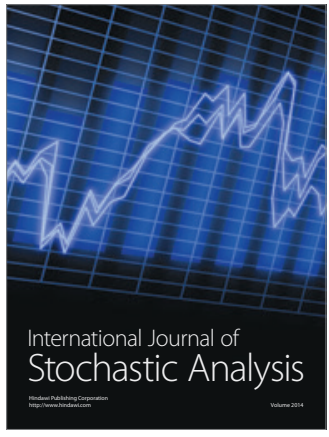

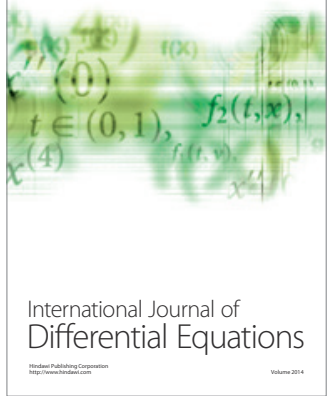
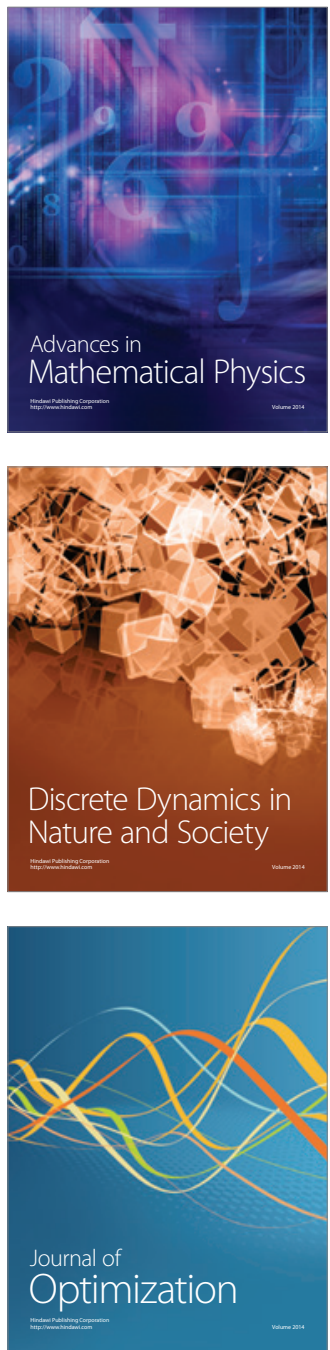